\title{
Consentimiento informado: síntesis de teoría actual y recomendaciones*
}

\author{
Drs. NICOLÁS ÁVALOS J. ${ }^{1}$, Abogado SANDRA TAPIA M. ${ }^{1}$ \\ 1 Comité de Ética Médica, Hospital de la Fuerza Aérea de Chile. \\ Santiago, Chile.
}

\begin{abstract}
\section{Recommendations and theoretical basis of the informed consent}

The informed consent is the rational acceptance of a medical intervention or the choice between several alternatives, by a patient. The intervention can be for diagnostic or therapeutic purposes. The concept of autonomous decision goes beyond the fulfillment of a legal regulation about the informed consent. We must not forget the ethical grounds of the relationship between health care providers and recipients, which should be based on mutual trust. The information should be disclosed in an honest, sincere and truthful way and without conflicts of interest, to obtain a fully informed consent to proceed with the proposed interventions.
\end{abstract}

Key words: Informed consent, provider, autonomy.

\section{Resumen}

El consentimiento informado es la aceptación racional por parte de un paciente de una intervención médica o la elección entre cursos alternativos posibles. Esta intervención puede ser de orden terapéutica o diagnóstica. El concepto de decisiones autónomas va así más allá del cumplimiento de una norma jurídica sobre consentimiento informado, por lo que no debemos perder de vista el fundamento ético de esta necesaria relación entre el profesional de la salud y el paciente, en un ámbito de confianza personal, de información otorgada en forma honesta, sincera, verdadera, de manera de obtener el consentimiento de éste para un determinado tratamiento.

Palabras clave: Consentimiento informado, actualidad, recomendaciones.

La frase consentimiento informado está retumbando en nuestros oídos desde ya hace algunos años, como aquel proceso de la relación médico paciente en que el primero informa al segundo, los procedimientos diagnósticos y/o terapéuticos que se le indican, sus riesgos, beneficios y alternativas, con el objeto de obtener su consentimiento, en forma previa, realizándose de esta manera el principio bioético y jurídico, de la autonomía del paciente. Sin embargo, aún existe cierta reticencia del profesional médico al momento de realizarlo y muchas veces se hace de una forma inadecuada.

*Recibido el 1 de abril de 2013 y aceptado para publicación el 12 de mayo de 2013.

Los autores no refieren conflictos de interés.

Correspondencia: Dr. Nicolás Ávalos J. Las Condes 8631, Santiago, Chile. contacto@cabezaycuello.cl 
En la experiencia hospitalaria, se ha vivido el esfuerzo de instalar la cultura del consentimiento informado. Hemos vivido varias etapas: desde el rechazo abierto a realizar este proceso a través de la suscripción de documentos, a la confección de documentos muy insuficientes, más como un documento de prueba, que como la muestra de una vivencia íntima, ética y libertaria del respeto a la persona humana.

Se ha pasado por las etapas de completar mecánicamente estos documentos, a veces parcial o imperfectamente, hasta instalarse este concepto a fuego dada la presión por la calidad, por la judicialización de la medicina, por el triunfo de la perspectiva de derechos de toda actividad humana, en el quehacer asistencial, en el léxico de los médicos y pacientes de nuestro país.

Se ha tratado de un esfuerzo por incorporar la necesidad y obligación de recoger en forma escrita un proceso que tradicionalmente y desde el punto de vista deontológico ha sido verbal, ello ha sido un camino desde la inseguridad y desconfianza por parte del estamento médico, hasta instalarse, sin lugar a dudas, como un cambio cultural importante en nuestra sociedad, que ha puesto al alcance de los pacientes mayor información, girando la relación médico paciente hacia una forma más horizontal de encontrarse, hacia un trato más digno, más humano y comprensivo de la naturaleza humana y del respeto a los derechos fundamentales, como la libertad, la autonomía, la dignidad humana.

El consentimiento informado llegó para quedarse, a pesar de todas las complicaciones surgidas en su aplicación e implementación, lo que necesariamente ha requerido un cambio por parte de todos los actores del proceso de atención de salud, médicos, profesionales de colaboración médica, pacientes, familiares, prestadores institucionales, llámese clínicas u hospitales públicos y privados.

Desde la mirada de la medicina, el consentimiento informado ha sido y sigue siendo parte del lex artis. Desde la perspectiva bioética, es la concreción del principio de la autonomía de la persona humana, sujeto del derecho a auto determinarse.

Desde hoy, ya es un proceso de carácter obligatorio, reconocido en varios cuerpos legales de nuestro ordenamiento jurídico nacional, tales como la recién promulgada y publicada Ley $\mathrm{N}^{\circ} 20.584$ sobre Derechos y Deberes del paciente y la Ley No 20.120 que lo reconoce con carácter obligatorio para todas las personas que participen en estudios clínicos o investigación científica de carácter médico.

El consentimiento informado es la aceptación racional por parte de un paciente de una intervención médica o la elección entre cursos alternativos posibles. Esta intervención puede ser de orden te- rapéutica o diagnóstica. El concepto de decisiones autónomas va así más allá del cumplimiento de una norma jurídica sobre consentimiento informado, por lo que no debemos perder de vista el fundamento ético de esta necesaria relación entre el profesional de la salud y el paciente, en un ámbito de confianza personal, de información otorgada en forma honesta, sincera, verdadera, de manera de obtener el consentimiento de éste para un determinado tratamiento.

Esta aceptación ha de otorgarse en forma libre, voluntaria y consciente; previa información otorgada por el médico sobre la naturaleza de su dolencia, sus riesgos y beneficios, así como de las alternativas posibles, con sus riesgos y beneficios, en un proceso que elementalmente es verbal y que, de acuerdo a la ley, sólo se escriturará en el caso de intervenciones quirúrgicas, procedimientos diagnósticos y terapéuticos invasivos y, en general, para la aplicación de procedimientos que conlleven un riesgo relevante y conocido para la salud del afectado. En estos casos, tanto la información misma, como el hecho de su entrega, la aceptación o el rechazo deberán constar por escrito en la ficha clínica del paciente y referirse al estado de su salud, del posible diagnóstico de su enfermedad, de las alternativas de tratamiento disponibles para su recuperación y de los riesgos que ello pueda representar, así como del pronóstico esperado, y del proceso previsible del postoperatorio cuando procediere, de acuerdo con su edad y condición personal y emocional.

El cumplimiento de esta obligación moral exige mejores estándares de atención en cuanto a la atención de salud, en cuento a tiempo, ambiente, lugares adecuados que garanticen la reserva de la información, derecho a las lágrimas, condiciones que los prestadores institucionales sean públicos o privados deben otorgar para el real ejercicio de este derechodeber, y en el ámbito público, exige un compromiso del Estado con el cumplimiento de estas básicas condiciones para que los profesionales de la salud puedan realizar este proceso informativo de forma respetuosa, íntima y reservadamente.

La necesidad de que los pacientes otorguen su aceptación a las intervenciones médicas a las que serán sometidos corresponde a la evolución del ser humano y de la sociedad frente al paternalismo que por centurias determinó el quehacer médico, en una relación de poder y dominación. El derecho a consentir o no, se funda en el respeto al principio de la autonomía de la voluntad de las personas, que nos dice que el individuo es dueño y responsable de su cuerpo y mente y que por lo tanto, es él quien en términos teóricos debe tomar responsabilidad y determinación con respecto a su salud.

No debe confundirse el consentimiento informado con una actitud reduccionista al considerarlo sólo 
un instrumento miope de la denominada medicina defensiva (sobretodo en la mirada médico legal). Es evidente, por tanto, que el consentimiento informado no puede reducirse a una recitación mecánica de hechos estadísticos o datos, ni a la firma de un formulario de autorización legal. El derecho a ser informado y dar la propia aceptación al acto médico que se va a realizar, es en sí mismo, un imperativo ético.

La Ley N $\mathrm{N}^{\circ} 20.584$ sobre Derechos y Deberes de los pacientes, ha reconocido el derecho a la información del paciente como un derecho importantísimo en la relación sanitaria, tanto frente a prestadores individuales, como a los prestadores institucionales, clínicas u hospitales, públicos o privados, y en este contexto se inserta el derecho al consentimiento informado, no sólo como el derecho a aceptar o rechazar un tratamiento, sino que a recibir información completa sobre sus dolencias, tratamientos, riesgos, beneficios etc., ello implica tiempo, atención sobre el paciente y sus emociones. Se trata de un paciente informado sobre terapias, aranceles, formas de pago, condiciones de ingreso, etc.

De la misma manera debemos aceptar como una alternativa dentro del proceso informativo, el rechazo a la terapia ofrecida. Si un paciente está bien informado y se le explica la evolución de la enfermedad con o sin terapia; puede libremente optar por no tratarse y no debe haber rechazo por parte del médico debido a esta opción, sino que una actitud abierta frente a esa posibilidad. En caso de duda razonable frente al mayor Bien el profesional deberá apoyarse en el Comité de Ética Médica que corresponda. Pero si el proceso de consentimiento ha sido bien desarrollado, es decir, con información adecuada, con lenguaje adecuado, explicando las consecuencias de su decisión y en paciente competente, el rechazo de tratamiento es una alternativa que dispone el paciente; y al tomarla no debe ser enviado al psiquiatra como todavía vemos hoy con cierta frecuencia.

El consentimiento informado es un proceso dialógico, que comienza desde la primera consulta médica. Durante la evaluación y estudio se va afianzando la relación médico paciente y esto se refuerza aún más cuando el paciente aprecia el esfuerzo médico de explicarle la causa de su dolencia y las posibles vías de solución. A esto se agrega la expresión de posibles complicaciones durante la evolución que pueden ser originadas en la misma enfermedad o por la terapia. Todo este proceso es llevado en última instancia a un documento escrito, el cual sólo es la "consagración" final de todo el proceso descrito y certificará que el proceso se realizó.

El consentimiento informado es un proceso y es un acto médico más, aunque el legislador haya tenido que reglamentarlos para obtener su exigibilidad y concreto ejercicio, y debe cumplir con algunas condiciones: Este derecho debe ser ejercido en forma libre, voluntaria, expresa e informada, para lo cual será necesario que el profesional tratante entregue información adecuada, suficiente y comprensible. En ningún caso el rechazo a tratamientos podrá tener como objetivo la aceleración artificial de la muerte, la realización de prácticas eutanásicas o el auxilio al suicidio; un estado de salud que permita al paciente recibir directamente la información y comprenderla, no existiendo por ende alteración de conciencia que afecte las condiciones anteriores, y en caso que ello ocurra, podrá ser informado y manifestar su aceptación o rechazo su representante legal, es decir, debe ser competente para tomar la decisión en cuestión.

El consentimiento informado es un proceso y es un acto médico más, el cual debe cumplir con algunas condiciones:

1. Disponer de la información adecuada por parte del paciente.

2. Comprender la información adecuadamente.

3. Encontrarse libre para decidir de acuerdo con sus propios valores.

4. Ser competente para tomar la decisión en cuestión.

Un consentimiento informado dentro de una institución no puede ser de carácter general y para todo evento al momento de hospitalización, como se estila una vez más, en la medicina defensiva. Incluso un documento de esta forma y de orden general carece de la fuerza legal suficiente en caso de alguna problemática de esa índole.

Como dijimos no sirve cualquier texto o documento como información básica para recoger la aceptación o rechazo del paciente, éste debe ser específico para la actuación propuesta, informar de forma suficiente, comprensible, para lo que podrá utilizar explicaciones verbales y escritas, dibujos, signos, símbolos etc., obtenerse de forma voluntaria y adaptarse a las condiciones básicas señaladas en la ley $\mathrm{N}^{\circ} 20.584$, ya citada.

El consentimiento debe obtenerse de manera voluntaria. Esta voluntariedad se debilita frente a actitudes, tales como: persuasión: que puede darse en forma no deliberada por parte del médico, o en forma implícita por el efecto psicológico del médico y su conocimiento; coacción: situación que puede suscitarse como amenaza implícita o explícita de pérdida de beneficios y derechos; manipulación: que puede darse al entregar información sesgada y/o distorsionada.

Estos agravios a la voluntariedad del consentimiento informado pueden ser más frecuentes de lo 
deseable, en actitudes tales como: "Firme aquí o no le doy tratamiento"; "Firme aquí o no lo opero" (en la puerta de pabellón). Esto hace perder absolutamente la legitimidad del consentimiento informado y atenta contra una adecuada relación médico-paciente. Un concepto importante que no hay que olvidar es el "tiempo necesario para una justa reflexión"; de esta forma el presionar por tiempo elimina el carácter de voluntario del consentimiento informado y por tanto, lo anula.

Un punto crítico a considerar es la entrega de información. Lo informado debe ser en cantidad suficiente para que el paciente pueda tomar una decisión. A su vez esta información debe ser de calidad adecuada al paciente, es decir, que sea comprensible para él en términos de lenguaje y complejidad. Hay que tener precaución frente al exceso de información que puede confundir al paciente, generar angustia patológica o llevarlo incluso a la desesperación.

La entrega de información no puede transformarse en una recitación despiadada de datos de sobrevida, pronóstico, riesgo u otras estadísticas. Todos los individuos son distintos. Existen limitaciones frente a la comprensión de la información otorgada que van desde el orden intelectual (educación), psicológicas-culturales (etnia, religión) y emocionales (el enfermo necesita integrar la información en su mundo frágil e inestable, como este evento impacta su biografía, proyectos y su forma particular de aproximarse a la verdad). El médico puede suministrar una amplia gama de información, pero no toda ella es necesaria para el consentimiento. Cuando un paciente aprehende su diagnóstico, muchas veces puede haber un momento de shock, hay un impacto en la vida de ese paciente que va desde su forma de ganarse la vida, hasta relaciones interpersonales y afectividad y así consolidarse como un quiebre en la biografía del sujeto. El paciente se presenta con una velada voluntad de conocer, que es variable, y que el médico debe descubrir para entregar la información de manera que su interlocutor pueda elaborarla. Se trata de que el enfermo escoja de alguna manera el ritmo y el límite de su descubrimiento y el médico, siempre detrás del enfermo, sin querer adelantársele, escoja adecuadamente el contenido y la forma en cada momento.

Muchas veces en una primera entrevista el paciente se enfrenta al shock del diagnóstico y luego no alcanza a elaborar y comprender la información que le da su médico para tomar una decisión adecuada para su tratamiento. Esto hace recomendar el entregar información escrita y tener una segunda entrevista previa al evento terapéutico. Esto tiene graduaciones según la gravedad del diagnóstico inicial (desde pueril a riesgo vital) y en cuanto a la celeridad en que deben tomarse las medidas terapéu- ticas (medidas de urgencia frente a un riesgo alto). Aquí el médico debe dar espacio para preguntas y sobretodo espacio para escuchar.

El grado de competencia de un individuo ha sido materia de importante estudio y sus consideraciones psiquiátricas, educacionales-culturales, neurológicas y legales pueden ser considerablemente distintas. La capacidad de comprensión puede variar enormemente de persona a persona y en distintas situaciones. El grado de competencia es una evaluación médica y es dinámica, es decir, variar en el tiempo. La capacidad o incapacidad es evaluada por el juez en un proceso legal como se explica más adelante.

Cuando la persona se encuentra en incapacidad de manifestar su voluntad y no es posible obtenerla de su representante legal, por no existir o por no ser habido, se adoptarán las medidas apropiadas en orden a garantizar la protección de la vida. Existen escenarios donde identificar el representante legal es muy difícil; ya que son los padres en los menores de edad y los nombrados por el juez en el incapaz. Pero en caso de un paciente adulto valente, inconsciente en ese momento, puede llegar a ser bastante difícil ya que puede no ser necesarariamente la esposa o el hijo mayor.

La capacidad desde el punto de vista legal es el primer requisito que debe concurrir en todo acto o contrato (art. 1.445 del Código Civil). La capacidad es la regla general y la incapacidad lo excepcional (art. 1.446).

De la falta de capacidad trata el art. 1.447 del citado Código, distinguiéndose entre los absolutamente incapaces y los relativamente incapaces.

Son absolutamente incapaces los dementes, los impúberes y los sordos o sordomudos que no pueden darse a entender claramente. Bajo ninguna circunstancia pueden actuar personalmente en la vida jurídica, sino sólo a través de representantes.

Son relativamente incapaces los menores adultos y los disipadores que se hallen bajo interdicción de administrar lo suyo. Los incapaces relativos deben actuar representados o personalmente si son debidamente autorizados.

El art. 43 del citado Código, establece quiénes son los representantes legales de una persona, el padre o la madre, el adoptante y su tutor o curador.

Cada vez que se inicia una entrevista que conlleva diagnósticos transcendentes y actos médicos acordes, se debe tener en mente el grado de competencia del enfermo en cuestión; para ese momento en particular (ejemplo: shock por noticia, psicosis aguda, menor de edad, etc.) y para ese diagnóstico en particular (desde tomar una aspirina a una amputación mayor como una laringectomía). Caso particular es el menor de edad con sus implicaciones legales. 
Frente a la menor duda que pudiera existir alguna merma en el nivel de competencia del individuo, se recomienda la asistencia de familiares cercanos en la entrevista para recalcar lo importante de la información otorgada y la trascendencia de las decisiones a tomar posteriormente. En caso de duda formal debe realizarse una interconsulta neurológica/psiquiátrica para evaluar formalmente la competencia.

Una situación en que recomendamos prestar especial atención es en el adulto mayor por dos consideraciones importantes: primero su situación de capacidad puede variar muy rápidamente en el tiempo (ejemplo: estado confusional postoperatorio) y segundo puede ser "incapaz en lo económico", es decir, son los hijos los que cubren sus gastos médicos y frente a complicaciones o extensión del tratamiento ser ellos la fuente de conflicto a pesar de la decisión consentida del padre (lamentablemente este sí es un consejo proteccionista). En estos casos recomendamos hacer parte del proceso de consentimiento a la familia extendida o al menos a uno de los hijos.

Son situaciones excepcionales en que la toma del consentimiento no es obligatorio:

1. Situación urgencia: En aquellos casos en que la condición de salud o cuadro clínico de la persona implique riesgo vital o secuela funcional grave de no mediar atención médica inmediata e impostergable y el paciente no se encuentre en condiciones de expresar su voluntad ni sea posible obtener el consentimiento de su representante legal, de su apoderado o de la persona a cuyo cuidado se encuentre, según corresponda, el tiempo obliga a actuar al médico. Incluye aquí el evento inesperado en el seno de otra intervención programada.

2. En el caso de que la falta de aplicación de los procedimientos, tratamientos o intervenciones señalados en el artículo anterior supongan un riesgo para la salud pública, de conformidad con lo dispuesto en la ley, debiendo dejarse constancia de ello en la ficha clínica de la persona.

3. Incompetencia del paciente: Cuando la persona se encuentra en incapacidad de manifestar su voluntad y no es posible obtenerla de su representante legal, por no existir o por no ser habido. En estos casos se adoptarán las medidas apropiadas en orden a garantizar la protección de la vida. Una alteración en el nivel de competencia de un paciente puede ser de carácter transitorio, esta situación debiera quedar acreditada con testigos.

4. Privilegio terapéutico: Privilegio del médico a tratar ya que la sola información puede perjudicar al paciente. Hoy en día es sólo excepcional.

5. Imperativo legal: Tratamientos, exámenes o vacunas impuestos por ley.
6. Rechazo expreso por parte del paciente: El paciente puede rechazar tener información y que las decisiones sean tomadas por otros. Debe quedar constancia explícita y escrita.

El derecho al consentimiento informado es parte integrante del derecho a la salud, consagrado en numerosos tratados internacionales de derechos humanos, con jerarquía constitucional. El consentimiento informado es un instrumento que sirve para proteger el derecho del paciente a participar en la adopción de decisiones sobre su salud, a la vez que genera obligaciones para los profesionales tratantes.

Creemos importante aquí destacar las Voluntades Anticipadas. Pacientes portadores de enfermedades crónicas que evolucionan hacia un desenlace fatal (ej: EBOC, Enfermedad de Alzheimer) o pacientes añosos que fácilmente pueden caer en situación de incompetencia (ej: paciente octogenario) facilita el proceso de cuidado de salud respetando los deseos del paciente al nombrar un representante y al dejar constancia de sus deseos (ej: no conectar a ventilación mecánica). Esto no sólo facilita el trabajo médico, sino que quita el gran peso de la responsabilidad a los hijos frente a decisiones muchas veces complejas. Los médicos que atendemos estos pacientes debemos ser promotores de las voluntades anticipadas como documento notarial para que tenga peso legal.

No resulta difícil percibir cómo la relación tradicional médico-paciente se ve entrelazada en las imágenes de nuestro tiempo y alimentada a la vez por los tecnicismos legales modernos. Las intervenciones médicas han generado nutrida discusión bioética en el mundo y afortunadamente se ha iniciado ya en Chile. El enfoque de valores y de derechos es la óptica correcta para recibir y dar sentido profundo a las nuevas normas legales que centran al paciente en el eje de la relación médico paciente, y sanitaria en general, (Ley N $\mathrm{N}^{\circ} 20.584$, sobre Derechos y Deberes de los pacientes en relación a su atención de salud), como una consecuencia más de la evolución política y social de nuestra sociedad, para ser mejores y más felices.

\section{Referencias}

1.- Beauchamp T, Childress JF. Respect for Autonomy. En: Principles of Biomedical Ethics, 6th ed. Oxford University Press, 2009;99-140.

2.- Jonsen A, Siegler M, Winslade W. Preferences of Patients. En: Clinical Ethics: a practical approach to ethical decisions in clinical medicine, 6th ed. McGraw-Hill Companies 2006;51-108.

3.- Kottow M. Encuentro clínico. En: Introducción a la 
bioética, $2^{\text {a }}$ ed. Editorial Mediterráneo 2005:225-35.

4.- Simon P, Diez mitos en torno al consentimiento informado. An Sist Sanit Navar. 2006;29 (Supl. 3):29-40.

5.- Monzón J, Saralegui I, Abizanda R, Cabré L, Iribarren $\mathrm{S}$, Martin M, y cols. Recomendaciones de tratamiento al final de la vida del paciente crítico. Med Intensiva 2008;32:121-33.

6.- Hernando P. Los derechos de los pacientes: una cuestión de calidad. Rev Calidad Asistencial 2005;20:353-6.

7.- Simon P, Judez J. Consentimiento Informado. Med Clin. (Barc) 2001;117:99-106.

8.- Tunzi M. Can the patient decide? Evaluating patient capacity in practice. Am Fam Physician 2001;64:299306.

9.- Simon P, Rodríguez J, Martínez A, López R, Judez J. La capacidad de los pacientes para tomar decisiones. Med Clin. (Barc) 2001;117:419-26.

10.- Bórquez G, Raineri G, Bravo M. La evaluación de la capacidad de la persona: en la práctica actual y en el contexto del consentimiento informado. Rev Med Chile 2004;132:1243-8.

11.- Ley No 20.120 República de Chile, (consultado el 21 de marzo de 2013) http://www.leychile.cl/Navegar? idNorma $=253478$.

12.- Ley $\mathrm{N}^{\circ} 20.584$ República de Chile, (consultado el 21 de marzo de 2013) http://www.leychile.cl/Navegar? idLey $=20584$.

13.- Código Civil República de Chile (consultado el 21 de marzo de 2013) http://www.leychile.cl/Navegar?idNor ma $=172986 \&$ idParte $=8717776$. 